\title{
Predictors of early and late outcome of percutaneous coronary intervention in octogenarians
}

\author{
Olivier GACH, Olivier LOUIS, Christophe MARTINEZ, Bernard LAMBERMONT ${ }^{\mathbf{1}}$, \\ Paul GERARD ${ }^{2}$, Victor LEGRAND \\ Division of Cardiology, University Hospital, Liège, Belgium; ${ }^{1}$ Department of Medicine, \\ University Hospital, Liège, Belgium; ${ }^{2}$ Department of Statistics, University of Liège, Belgium.
}

Objective - To evaluate the short and long-term results of percutaneous coronary interventions $(\mathrm{PCl})$ in patients aged 80 years or older and to identify predictors of event-free survival.

Methods and results - Clinical and angiographic data from all patients undergoing percutaneous coronary intervention in our institution are prospectively collected and stored in a computerized database. The clinical and angiographic characteristics of all patients aged 80 years or older undergoing percutaneous coronary intervention between January 1994 and December 1999 were analysed retrospectively. Follow-up was obtained by interview or through the referring physician. One hundred and fifty-eight patients aged 80 years or older (median: 83.4; range: 80.2-92.2) underwent percutaneous coronary intervention in our institution during the study period. The initial angiographic success rate was $92 \%$. In-hospital mortality was $8.2 \%$ and procedural success $84.8 \%$. One-year and two-year survival were $81 \%$ and $72.2 \%$ respectively, while event free survival at I year and 2 years was $65.8 \%$ and $57 \%$. Using the Cox proportional hazards method, we identified incomplete revascularization and low left ventricular ejection fraction (LVEF) as predictors of death at 2 years. Complete revascularization and stenting were independent predictors of 2-year event-free survival.

Conclusion - Percutaneous coronary intervention can be performed safely in octogenarians. Complete revascularization, stenting and preserved left ventricular ejection fraction were independent predictors of better outcome in this population. (Acta Cardiol 2003; 58(4): 289-294)

Keywords: coronary intervention - octogenarians - revascularization - angioplasty.

\section{Introduction}

Very elderly people ( $>80$ years) comprise the fastest growing segment of our population and in these patients, cardiovascular disease represents the leading cause of morbidity and mortality ${ }^{1}$. Therefore, an increasing number of elderly patients with symptomatic coronary artery disease is considered for myocardial revascularization. Since coronary artery by-pass grafting $(\mathrm{CABG})$ in the geriatric population is associated with increased morbidity and mortality ${ }^{2}$, percutaneous coronary intervention (PCI), a less invasive procedure, may be more attractive. Nevertheless, there are limited data on efficacy and safety of PCI in octogenarians;

Address for correspondence: Victor Legrand, M.D., Division of Cardiology, University Hospital of Liège, B - 4000 Liège, Belgium. Tel.: +32 436671 92. Fax: +32 436678 37. E-mail: vlegrand@chu.ulg.ac.be Received 8 October 2002; revision accepted February 26, 2003. therapeutic decision-making is made more difficult because the very elderly are underrepresented in randomized trials.

This study describes acute and 2-year follow-up results of PCI in the subset of patients aged 80 years or older in our institution over a 6-year period.

\section{Methods \\ PATIENTS}

The study population included 158 consecutive patients aged 80 years or above who underwent PCI in our institution between 1 January 1994 and 31 December 1999. Baseline in-hospital data were obtained from a computerized database of prospectively recorded demographic, clinical and procedural information.

One and two-year clinical follow-up information was obtained from the referring physician or by inter- 
views with the patient. Information on vital signs, current angina status and occurrence of cardiac events (defined as death, myocardial infarction (MI), CABG, or repeat PCI) were recorded. The indications for repeat coronary angiography were strictly clinical. Complete follow-up data were available for all patients.

The analysis of clinical, procedural, angiographic, in-hospital and late clinical events comprised the basis of this report.

\section{Coronary intervention procedure}

Angioplasty was performed using the femoral route. All patients received oral aspirin; those who underwent coronary stenting also took ticlopidine for 1 month. No patient received Gp IIb/IIIa inhibitors. There was a gradual increase in stent utilisation during the study period. No stents were implanted in procedures performed in 1994, while they were used in $70.2 \%$ of the procedures done in 1999.

\section{Clinical definitions}

Procedure priority was defined as urgent if the PCI was required within 24 hours of presentation due to either unstable angina refractory to medical therapy; ongoing myocardial ischaemia, or cardiogenic shock. All other procedures were considered as elective.

In-hospital mortality was defined as the occurrence of death during the post-procedural phase.

Myocardial infarction (MI) was defined as the development of new $\mathrm{Q}$ waves in 2 or more contiguous leads on a12-lead electrocardiogram taken in the hospital with a rise in serum CK-MB (greater than three times normal).

Vascular complications included: entry site occlusion, groin haematoma, documented pseudoaneurysm, entry site bleeding requiring blood transfusion or surgical repair, dissection of iliac or femoral arteries or acute limb ischaemia.

Stroke was considered when a permanent loss of neurologic function caused by an ischaemic vascular event occurred.

Cardiogenic shock was established when systolic blood pressure remained $<90 \mathrm{~mm} \mathrm{Hg}$ without inotropes or intra-aortic balloon pump insertion, or when pump failure was noted, as manifested by a cardiac index $<2.2 \mathrm{l} / \mathrm{min}$ per $\mathrm{m}^{2}$ and pulmonary capillary wedge pressure $>18 \mathrm{~mm} \mathrm{Hg}$. Persistence of hypotension or pump failure after correction of contributing extramyocardial factors and/or peripheral signs of hypoperfusion were also required for this diagnosis.

Angiographic success was defined as $<50 \%$ residual diameter stenosis and $<30 \%$ after stenting at the dilatation site with normal TIMI 3 coronary flow.
Procedural success was defined as angiographic success without no major complications (defined as death, MI, or emergency repeat revascularization) at any time during hospitalisation.

Complete revascularization was defined as successful dilatation of all stenoses of $>50 \%$ luminal diameter.

Repeat revascularization was defined as the need for either CABG or PCI after the index intervention.

\section{STATISTICAL ANALYSIS}

The effect on survival and event-free survival of the covariates listed in table 1 was investigated through the Cox proportional hazards method ${ }^{3}$. First, a univariate Cox regression analysis was applied to all covariates. Then, the selected covariates $(\mathrm{p}<0.2)$ were analysed by a backward procedure using a multivariate Cox regression. $\mathrm{P}<0.05$ was considered significant. Life-table analyses were performed by the Kaplan-Meier method ${ }^{4}$. Time to events for survival analyses were measured from the index catheterization.

\section{Results}

\section{CLINICAL CHARACTERISTICS}

From 1 January 1994 to 31 December1999, 158 patients aged 80 years or older (mean: 83.4; range: 80.292.2) underwent PCI in our institution. These patients (54.8\% men) represent $2.36 \%$ of the 6,692 patients treated in the same institution during this period. Clinical and angiographic characteristics of this population are shown in table 1 . As other authors previously report $^{5}$, women account for more than $40 \%$ of this elderly population who have higher prevalence of hypertension. Prior myocardial infarction (MI) was noted in $30 \%$ of these octogenarians, previous PCI in $13 \%$, and previous CABG in $11 \%$. Nearly $70 \%$ had multivessel disease. Left ventricular ejection fraction was conserved, but left ventricular end diastolic pressure (LVEDP) was $23 \pm 8 \mathrm{~mm} \mathrm{Hg}$. Two hundred and five lesions were treated (1.3 per procedure); although $67 \%$ of the patients had multivessel disease, revascularization was restricted to one vessel in 114 patients and two or more in 44 patients $(28 \%)$. Revascularization was complete in $49.4 \%$. Angioplasty was urgent in 120 patients $(76 \%)$ and elective in 38 patients $(24 \%)$. Implantation of stents was performed during 69 procedures $(43.7 \%)$.

\section{IN-HOSPITAL OUTCOME}

Angiographic success was $92 \%$ (failure to cross the lesion in five cases, abrupt closure of ten lesions, and 
Table 1. - Baseline clinical and angiographic characteristics of the population

\begin{tabular}{lc}
\hline Number of patients & 158 \\
Age*(years) & 83.4 \\
Men* (\%) & $86(54.8)$ \\
Hypertension (\%) & $111(70.2)$ \\
Hypercholesterolaemia* (\%) & $68(43)$ \\
Diabetes* (\%) & $29(18.3)$ \\
Smoking history* (\%) & $62(39.2)$ \\
Family history* (\%) & $23(14.6)$ \\
Previous myocardial infarction* (\%) & $44(30.4)$ \\
Previous PCI* (\%) & $21(13.3)$ \\
Previous CABG* (\%) & $18(11.4)$ \\
Urgent procedure* (\%) & $120(76)$ \\
Unstable angina (\%) & $78(49.4)$ \\
Post-myocardial infarction angina (\%) & $26(16.5)$ \\
Cardiogenic shock (\%) & $8(5)$ \\
Acute myocardial infarction (\%) & $8(5)$ \\
Stent* (\%) & $69(43.7)$ \\
Multivessel disease* (\%) & $106(67)$ \\
Main left disease* (\%) & $9(5.7)$ \\
Multi PCI (\%) & $44(27.9)$ \\
Complete revascularization (\%) & $78(49.4)$ \\
Restenotic lesion* (\%) & $11(5.4)$ \\
Left anterior descending artery PCI* (\%) & $89(43.4)$ \\
Circumflex artery PCI* (\%) & $42(20.5)$ \\
Right coronary artery PCI* (\%) & $64(31.2)$ \\
Ejection fraction* & $58.3 \pm 16.2 \%$ \\
LVEDP* (mm Hg) & $23 \pm 7.9$ \\
\hline
\end{tabular}

CABG: coronary artery by pass graft; LVEDP: left ventricular end diastolic pressure; PCI: percutaneous coronary intervention.

* Variables analysed in the statistical model.

no reflow in one case). In-hospital mortality was $8.2 \%$ (13 patients). Four patients died of cardiogenic shock after angiographic failure. Nine patients died despite angiographic success (cardiogenic shock in five, renal failure after surgical repair of a pseudoaneurysm in one, heart failure in two and stroke in one). Seven patients developed non-fatal MI, including two non-Q wave MI; four further patients underwent emergency CABG because of failed angioplasty. Overall procedural success was obtained in 134 patients $(84.8 \%)$. Six patients $(3.8 \%)$ developed vascular complications after the procedure. These included pseudoaneurysm formation $(n=2)$ and groin haematoma $(n=4)$. Three patients required surgical repair of the femoral artery and one required blood transfusion.

\section{LATE CARDIAC EVENTS}

Two-year follow-up was available for all patients. During the first year of follow-up, 30 patients died including 13 in-hospital deaths. During the second year, 14 additional patients died (mortality at two years: $27.8 \%$ ), representing an actuarial survival at two years of $72.2 \%$ (Figure 1 ). The causes of out-of-hospital

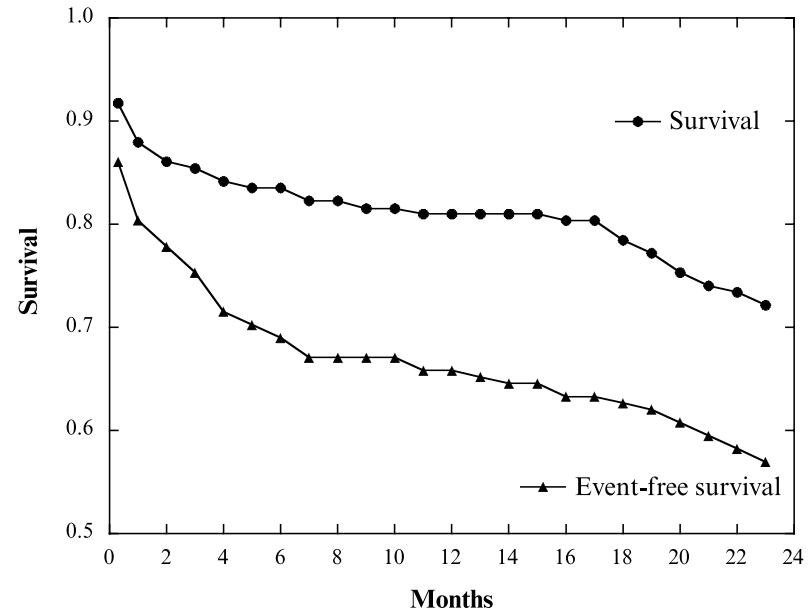

Fig. 1. - Actuarial global and event-free survival.

$\longrightarrow-$ : Actuarial global survival of the population.

$\_$: Actuarial event-free survival of the population.

deaths were unknown and presumed to be cardiac in 12 cases [sudden death $(n=6)$, heart failure $(n=4)$, fatal MI $(n=2)]$. Fatal stroke, respiratory insufficiency and carcinoma were the causes of death in one, two and four patients, respectively. After hospital discharge, 17 patients underwent repeat revascularization $(11.7 \%)$. This was obtained by repeat angioplasty in 15 and surgery for the two others. None suffered non-fatal out-of-hospital MI. The event-free survival (absence of death, MI or repeat revascularization) at one and two years was, $65.8 \%$ and $57 \%$ respectively. At two years, 14 additional patients died of whom two were revascularized during the first year of follow-up and two required later repeat PCI. Figure 1 shows global actuarial survival and event-free survival at two years by the Kaplan-Meier method.

\section{Predictors of outcome (TABle 2)}

Using the Cox proportional hazards method, predictors of mortality at two years were incomplete revascularization $(p=0.032)$ and low left ventricle ejection fraction $(\mathrm{LVEF})(\mathrm{p}=0.004)$. Predictive factors of

Table 2. - Predictors of outcome (Cox proportional hazards analysis)

\begin{tabular}{lcc}
\hline & $\begin{array}{c}2 \text { year } \\
\text { mortality }\end{array}$ & $\begin{array}{c}2 \text { year } \\
\text { event free }\end{array}$ \\
\hline Complete revascularization & $\mathrm{p}=0.032$ & $\mathrm{p}=0.002$ \\
& OR: $0.51 \pm 0.32$ & OR: $0.45 \pm 0.25$ \\
Ejection fraction (\%) & $\mathrm{p}=0.004$ & $\mathrm{NS}$ \\
OR: $0.97 \pm 0.01$ & \\
Stent & $\mathrm{NS}$ & $\mathrm{p}=0.024$ \\
& & OR: $0.56 \pm 0.26$ \\
\hline
\end{tabular}

NS: non-significant, OR: odds ratio. 


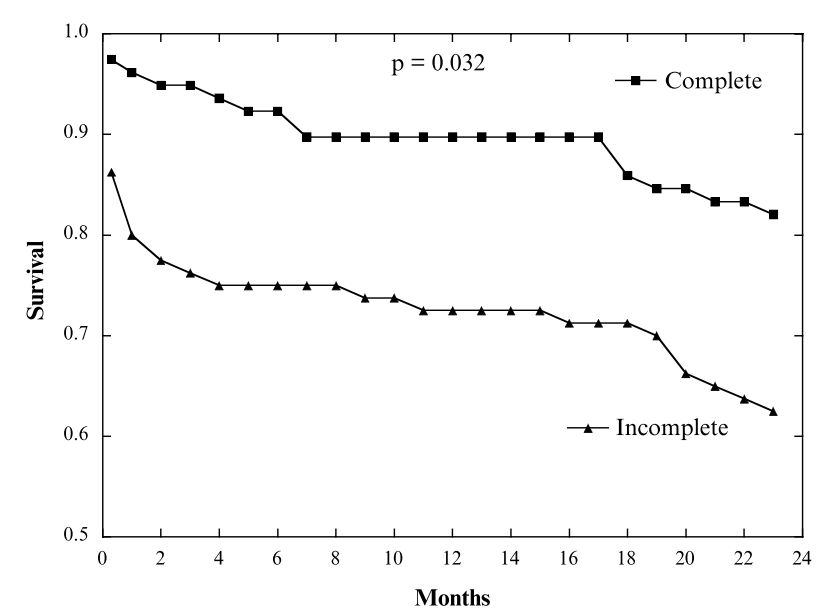

Fig. 2. - Influence of complete revascularization on survival. $\longrightarrow$ : Actuarial survival with complete revascularization.

event-free survival at two years were stent implantation $(p=0.024)$ and complete revascularization $(p=0.002)$. The influence of complete revascularization and stent use on mortality and on event-free survival is shown in figures 2 and 3.

\section{Discussion}

The proportion of elderly patients suffering coronary artery disease continues to increase as a result of the increasing average life expectancy. This explains the growing number of revascularization interventions in the aged ${ }^{6-8}$. Despite the fact that octogenarians are considered to be a high-risk group with an increased rate of procedure-related complications, the need for revascularization in symptomatic patients is established. On the other hand, the choice of therapeutic modality is difficult, because these patients are underrepresented in randomized trials and there are few data on late outcome in this subgroup.

\section{SURVIVAL}

In this study, percutaneous revascularization during which stents were implanted in only $43.7 \%$ of patients, achieved angiographic success in $92 \%$ and procedural success in $84.8 \%$. This is comparable to other reports analysing only in-hospital results, and in which stents were implanted in $40 \%$ of the procedures ${ }^{9}$.

Our in-hospital mortality of $8.2 \%$ was higher than in the NCN report ${ }^{9}$, probably due to the high number of emergency interventions in our study $(76 \%)$. As in the younger population, low LVEF and urgent procedure adversely affect survival. The completeness of revascularization appeared to be very important in this
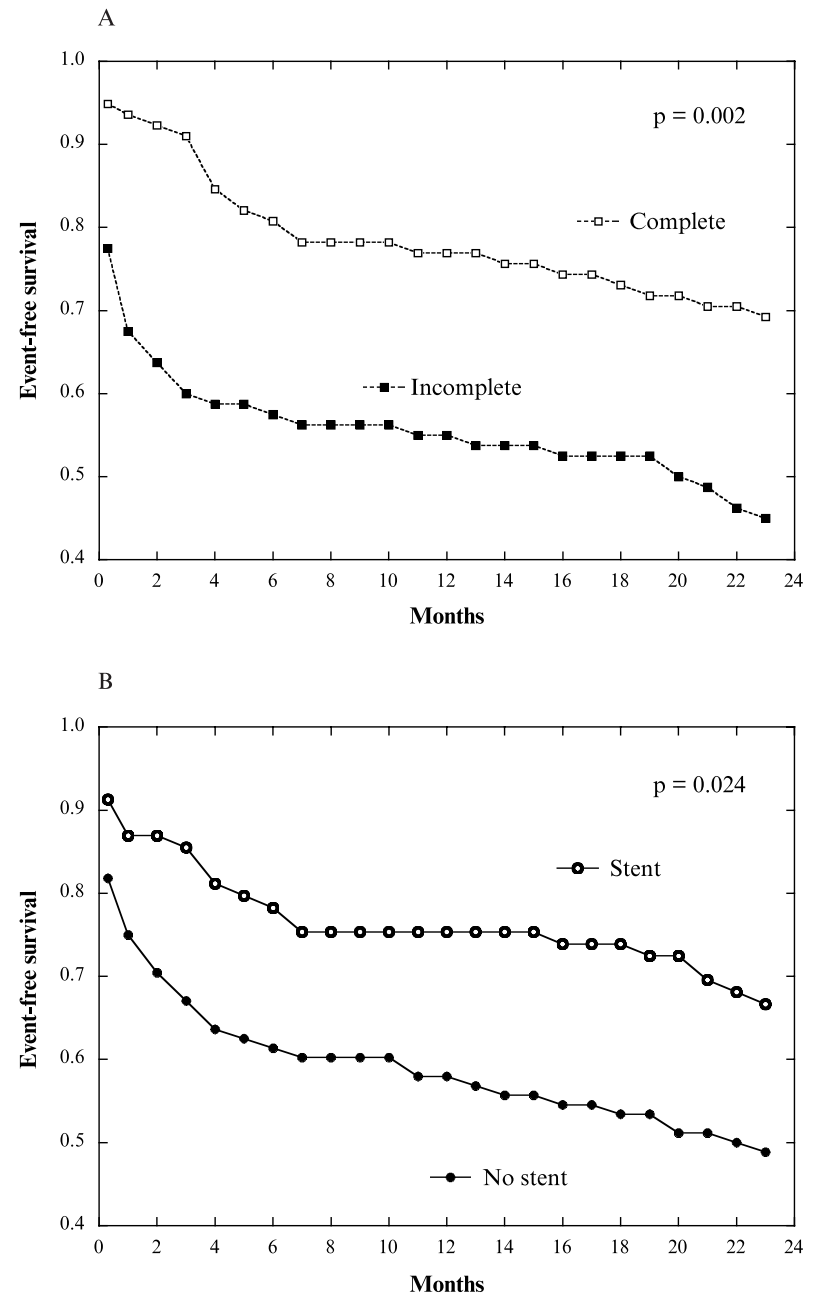

Fig. 3. - Influence of complete revascularization (3A) and stent (3B) on event-free survival.

-- -- : Actuarial event-free survival with complete revascularization.

-0- : Actuarial event-free survival: PTCA with stent.

-1 : Actuarial event-free survival: PTCA without stent.

--- : Actuarial event-free survival with incomplete revascularization.

study. The need to treat all significant lesions is underlined here, but has not been studied in previous series. Despite the fact that multivessel disease was present in $67 \%$ of patients, only $28 \%$ of the population had multiple vessel PCI and complete revascularization was achieved in only $49.4 \%$. These findings are similar to those of other authors ${ }^{10-13}$. All patients with single-vessel disease therefore benefit from complete revascularization. It is possible that the strong effect of complete revascularization could be due to selection bias, because of less extensive disease in this subgroup. Nevertheless, this would not appear to be the case because analysis of the effect of single-versus multivessel disease on outcome did not appear to be statistically significant. PCI was sometimes chosen for revascularization because the 
patient was considered to be at high risk for surgery or had haemodynamic instability. In the circumstances treatment of the culprit lesion was carried out in an attempt to improve symptoms without accomplishing complete revascularization, this reducing procedure time and the amount of contrast utilised. These precautions have nevertheless an important impact on prognosis. In terms of long-term results, low ejection fraction and complete revascularization are crucial determinants. For 1 and 2-year mortality, poor left ventricular systolic function was significantly associated with both lower procedural success and a trend towards a worse outcome. Similar results have been shown by other investigators ${ }^{14,15}$ but the impact of completeness of revascularization was not as strong. Nevertheless, only 44 patients died in our small study population. Therefore, non-selection of a variable does not conclusively reject a possible role in outcome, but may be secondary to a small sample size.

\section{Event-free survival}

Complete revascularization protects from death but also from secondary adverse events, while stent placement only prevents adverse events. The protective effect of stenting was also described in the NCN report for short-term results. Vascular complications (3.8\%) occurred with nearly the same frequency in other studies $5,16-19$, confirming that older patients and women continue to have a higher incidence of vascular complications compared to the younger population. This could be due in part to an increased risk for vascular injury because of underlying peripheral vascular disease, the prolonged half-life of heparin in elderly patients, or more aggressive anticoagulation in these patients. Gp IIb/IIIa inhibitors were not implicated because no patient in this study received these drugs. The recent EPISTENT ${ }^{20}$ trial did not, however, show increased bleeding with the use of abciximab. Nevertheless, given the higher rate of vascular complications in the elderly, the use of these agents in this population should be based on careful consideration of risk and benefit.

Completely revascularized and stented patients demonstrate better outcome with no association between these two factors. Other studies of stenting without balloon angioplasty reported better in-hospital and one year results but this population was analysed using data from six randomized trials with strict clinical and angiographic inclusion and exclusion criteria. Results therefore represent an ideal octogenarian population ${ }^{5}$, differences from our study probably reflect the healthier population enrolled in these investigations. Our heterogeneous populations with severe clinical presentations is likely to more closely represent daily clinical practice.

\section{Alternative TREATMENT}

Very large series have recently been published concerning acute results of surgical revascularization. They report in-hospital mortality of $8.1 \%$ but with a doubling of the incidence of postoperative stroke and renal failure in comparison with younger patients ${ }^{21}$. Longterm follow-up of such a large population is not yet available. A local study evaluating $\mathrm{CABG}$ results in octogenarians performed in the same institution during nearly the same period revealed an in-hospital mortality of $10 \%$ but a 5 -year mortality of $20 \%(14 / 70)^{22}$. Predictors of operative mortality were New York Heart Association functional class IV, prolonged extracorporeal circulation and urgent CABG (13\% of this surgical population). As concerns late outcome, previous MI and urgent procedure were predictive. No statistical evaluation was performed in order to compare these two local studies and therefore no conclusions can be drawn about similarities and differences between these results.

In a recent randomized trial ${ }^{23}$, medical treatment compared with PCI or CABG clearly demonstrated better clinical outcome and well-being after revascularization. Moreover, one third of the patients randomized to medical therapy required revascularization during follow-up for uncontrolled symptoms.

\section{Limitation of the study}

This study is a retrospective analysis of data which were collected prospectively and is subject to all the limitations inherent in such a study. It was not a randomized trial, and no attempt was made to compare results with $\mathrm{CABG}$ or medical therapy. The population reflects a relatively large non-selected, single-centre experience and provides insight into difficulties we had in offering optimal revascularization strategy to the elderly population.

The sample size was relatively limited. This may be partly responsible for the non-selection of some variables in this study.

\section{Conclusion}

Despite real progress in managing coronary insufficiency, CABG can be performed with an acceptable risk in selected patients, but with a higher incidence of in-hospital complications and mortality in the elderly when compared to younger patients. Several retrospective studies have also demonstrated the safety and efficacy of PCI, in selected patients, providing satisfactory symptom relief and favourable mid-term outcome. This study emphasises the beneficial effects of technical progress such as stent placement and the 
necessity of performing, when feasible, a complete revascularization in these high-risk patients in order to improve initial and late outcomes.

\section{References}

1. Specer G. U.S. Bureau of the Census: projections of the population of the United States, by age, sex and race: 1988 to 2080. Washington, DC: U.S. Government Printing Office, Current Population Reports, 1989, series P-25; n 1018.

2. Scheidt S. Risk stratification parameter in patient selection for coronary artery bypass grafting. Am J Cardiol 1999; 83: $3 b-9 b$.

3. Collett D. Modelling survival data. In: Collett D, Chatfield C, Zidek JV, ed. Modelling survival data in medical research. Chapman and Hall, London, 1984: 53-106.

4. Kaplan EL, Meier P. Nonparametric estimation from incomplete observations. J Am Stat Assoc 1958; 53: 457-81.

5. Chauhan MS, Kuntz RE, Ho KKL, Cohen DJ, Popma JJ, Carrozza JP, Baim DS, Cutlip DE. Coronary artery stenting in the aged. J Am Coll Cardiol 2001; 37: 856-62.

6 . Wenger NK. Coronary disease in elderly patients: myocardial infarction and myocardial revascularization. Heart Dis Stroke 1994; 3: 401-6.

7. Feinleib M, Havlik RJ, Gillum RF, Pokras R, McCarthy E, Moien M. Coronary heart disease and related procedure: national hospital discharge survey data. Circulation 1989; 79: I-113-118 (Suppl I).

8. Peterson ED, Jollis JG, Bebchuk JD, DeLong ER, Muhlbaier LH, Mark DB, Pryor DB. Changes in mortality after myocardial revascularization in the elderly. The national Medicare experience. Ann Intern Med 1994; 121: 919-27.

9. Batchelor WB, Anstrom KJ, Muhlbaier LH, Grosswald R, Weintraub WS, O’Neill WW, Peterson ED. Contemporary outcome trends in the elderly undergoing percutaneous coronary interventions: result in 7,472 octogenarians. $J$ Am Coll Cardiol 2000; 36: 723-30.

10. Abizaid AS, Popma JJ, Mehran R, Abizaid A, Clark CE, Pichard AD, Kent KM, Satler LF, Leon MB. Coronary stenting in the elderly: early in-hospital and long-term clinical outcomes. J Am Coll Cardiol 1998; 31: 234A (abstract suppl).

11. Tan KH, Sulke N, Taub N, Karani S, Sowton E. Percutaneous transluminal coronary angioplasty in patients 70 years of age or older: 12 years' experience. Br Heart J 1995; 74: 310-317.

12. Deligonul U, Vandormael MG, Kern MJ, Zelman R, Galan K, Chaitman BR. Coronary angioplasty: a therapeutic option for symptomatic patients with two and three vessel coronary disease. J Am Coll Cardiol 1988; 11: 1173-9.
13. Bell MR, Bailey KR, Reeder GS, Lapeyre AC, Holmes DR. Percutaneous transluminal angioplasty in patients with multivessel coronary disease: how important is complete revascularization for cardiac event-free survival ? J Am Coll Cardiol 1990; 16: 553-62.

14. Stevens T, Kahn JK, McCallister BD, Ligon RW, Spaude S, Rutherford BD, McConahay DR, Johnson WL, Giorgi LV, Shimshak TM. Safety and efficacy of percutaneous transluminal coronary angioplasty in patients with left ventricular dysfunction. Am J Cardiol 1991; 68: 313-9.

15. Serota H, Deligonul U, Lee WH, Aguirre F, Kein MJ, Taussig SA, Vandormael MG. Predictors of cardiac survival after percutaneous transluminal coronary angioplasty in patients with severe left ventricular dysfunction. Am J Cardiol 1991; 67: 367-72.

16. DeGregorio JD, Kobayashi Y, Albiero R. Coronary atery stenting in the elderly: short-term outcome and long term angiographic and clinical follow-up. J Am Coll Cardiol 1998; 32: $577-83$.

17. Batchelor WB, Peterson ED, Anstrom KJ, Muhlbaier LH, Grosswald RD. Does stenting benefit the very elderly? An analysis of device-specific outcomes in 3,470 octogenarians. J Am Coll Cardiol 1998; 31: 77A (abstract suppl).

18. Lindsay JJr, Reddy V, Pinnow EE, Little T, Pichard AD. Morbidity and mortality rates in elderly patients undergoing percutaneous coronary transluminal angioplasty. Am Heart $J$ 1994; 128: 697-702.

19. Leon MB, Baim DS, Popma JJ, Gordon PC, Cutlip DE, Ho KK, Giambartolomei A, Driver DJ, Lasorda DM, Williams DO, Pocock SJ, Kuntz RE. A clinical trial comparing three antithrombotic drug regimens after coronary artery stenting. $N$ Engl J Med 1998; 338: 1665-71.

20. EPISTENT investigators. Randomised placebo-controlled and ballon-angioplasty-controlled trial to assess safety of coronary stenting with use of platelet glycoprotein-IIb/IIIa blockade. Lancet 1998; 352: 87-92.

21. Alexander KP, Anstrom KJ, Muhlbaier LH, Grosswald RD, Smith KP, Jones RH, Peterson ED. Outcomes of cardiac surgery in patients aged $>80$ years: results from the national cardiovascular network. J Am Coll Cardiol 2000; 35: 731-8.

22. Kolh P, Kerzmann A, Lahaye L, Gerard P, Limet R. Cardiac surgery in octogenarians. Peri-operative outcome and longterm results. Eur Heart $J$ 2001; 22: 1235-43.

23. The TIME investigators. Trial of invasive versus medical therapy in elderly patients with chronic symptomatic coronaryartery disease (TIME): a randomised trial. Lancet 2001; 358: 951-7. 